\title{
Development of a Multipurpose Mobile Robot for Concrete Surface Processing
}

\author{
A Status Report \\ by \\ Rolf Berlin and Ferenc Weiczer \\ Department of Production Engineering, Robotics Laboratory \\ Chalmers University of Technology \\ Gothenburg, Sweden \\ February, 1992
}

\begin{abstract}
This paper reports on the continuing design of an autonomous multipurpose mobile robot intended for concrete slab finishing, grinding, and cleaning during construction. Common to these three applications are rotating tools in contact with the floor. Selectively adaptable to one machine, these tools will perform recurrent tasks on a construction site. In Phase 1, an experimental vehicle has been built to test navigation concepts including path planning, location measurement, and path following. Steering and locomotion of the experimental vehicle are provided by two independently steered and powered wheel sets enabling the vehicle to perform complex manoeuvres required by the selected application. In Phase 2, a robot prototype equipped with tools is under development. Different vehicle-tool configurations have been modelled and simulated in order to attain optimal mobility and surface coverage.
\end{abstract}

\section{Keywords:}

Autonomous Mobile Robot, Concrete Surface Processing, Path Planning, Location Measurement, Path Following, Vehicle Surface Interaction.

\section{INTRODUCTION}

Within the Swedish Construction Federation in Gothenburg, a group of ten Swedish contractors, called "FoU-Väst", has been managing, since 1987, a project about the use of automation and robotics in the construction industry. Also involved in the project are equipment manufacturers, the Labour Union and the Construction Industry's Organization for Work Environment Safety and Health, and the Robotics Laboratory at Chalmers University of Technology. 
Within the group a thorough analysis has been made of current methods used in Sweden. It showed that improvements, in the form of automation or use of robotics, were desirable in, for example, concrete-work, cleaning, masonry, and work at great heights. But more R\&D work has to be done before the intentions can be realized.

As a pilot project, construction of a mobile robot for concrete surface finishing, such as slab finishing, grinding, and cleaning, was started in 1990. In phase 1, those three applications were studied and a specification of the robot was defined. To evaluate navigation concepts and the onboard motion control system, a laboratory prototype was developed. In phase 2, a field test prototype with multitool capability is under development.

Besides indicating the need for automation the analysis also pointed up the occasionally long idle periods for a machine on a construction site. To increase the utilization of the robot a multipurpose machine is desirable. However, the multipurpose use of the robot increases the demands on mobility and compact design.

The following sections presents an overview of the ideas, hardware and software solutions, and a summary of the results so far. In section two, the architecture of the experimental vehicle is presented. In the third section, the structure of the onboard navigation system is outlined, including a short description of a rebar detector and a laser optical locating device. The fourth section describes the basic principles of the simulation of the robot motions. In section five, some results from recent simulations of various vehicle and tool configurations are discussed, focusing on vital design considerations.

\section{EXPERIMENTAL VEHICLE}

The purpose of the experimental vehicle is to act as a test-bed for evaluation of the onboard motion control system, and the locating and safety sensors [1]. The locomotion and the steering of the experimental vehicle are realized by two sets of independently driven and steered wheels. This configuration of dual steer, dual drive enables the vehicle to perform manoeuvres not possible for a tricycle or a differential type cart. The vehicle is equipped with a 24-volt battery power source. Front and rear wheels are powered by two DC motors of PM type. The steering motors are of the same type. Four rebar detectors are used to aid the measurement of vehicle location. The actual steering angles of front and rear wheels are measured by two incremental encoders with zero-pulse detection, mounted on the vertical axis of each wheel as shown in Fig. 1. The distances travelled by the wheels are calculated by counting pulses from incremental encoders mounted on the horizontal wheel axes. The actual velocities are determined from the same data by pulse-width measurement.

\section{NAVIGATION}

The problem of navigation is central to mobile robot applications. Beyond the level of mapping and modelling the environment, it may be divided into a number of subtasks: Task planning, path planning, location measurement, and path following. The task planner, specifies the destination and the constraints on the path, such as velocity, obstacles, etc. Given a suitable path from start to goal, generated by a path planner module, the final step is to run the vehicle along the path. During path following, deviations from the desired path are calculated by examining data from external or onboard sensors for location measurement. Once the path error is known, it has to be eliminated or minimized. 
Path planning means to determine a trajectory for a reference point on the vehicle while it is moving from a given start position to a desired destination. Algorithms generating surface coverage paths while taking into consideration tool overlap, obstacle avoidance, and processing efficiency, have been developed. The path is generated off-line, allowing the operator to modify path or tool parameters.

The location of the robot is calculated by the dead-reckoner module of the onboard vehicle controller from data provided by incremental encoders for steering angle and travelled distance measurement. Among locating devices, that may be used to reset the deadreckoner module, we find a number of devices such as laser optical systems, ultrasonic sensors, gyros, etc. [2],[3].

Our interest is focused on a new method utilizing a rebar detector for orientation measurement as well as a laser locating device. The reason for studying these two methods is the wish to gain experience with a new, robust and inexpensive sensor system as compared to a sophisticated and commercially available laser locating system.

\subsection{Location measurement with rebar detector}

A new sensor to supplement location measurement is under development. It senses the interference with a generated magnetic field caused by the presence of the rebars embedded in the concrete floor.

The four detectors, mounted on the front, the rear and the sides of the vehicle, are able to detect the rebars within a vertical sensing range of $150 \mathrm{~mm}$. These data are used to adjust the location estimate of the dead-reckoner module.

The example, in Fig. 2, illustrates how orientation of the vehicle can be calculated while it passes over a rebar. We assume that the vehicle moves along a straight line, not altering its heading between the instants of sampling a rebar.

\subsection{Location measurement with Laser sensor}

The laser unit uses eye-safe laser beams to scan the navigation area. A pulsed infrared laser is used to measure distance with the time-of-flight method [4]. No reflectors are needed for detection. The range of the instrument is $1-100 \mathrm{~m}$.

The sum of all points results in a digitalized image of the surroundings as shown in Fig 3. The laser system compensates for the robot's movement during a laser scan.

A map of the navigation area is created either by an operator using a CAD program or by the device itself scanning the area from a known location.

The output is a correction vector generated upon comparison of the map and the laser image, giving an accuracy of approx. $1 \mathrm{~cm}$ as long as at least $70 \%$ of the map is visible on the laser scan.

Besides location measurement, the laser range finder also provides obstacle detection, eliminating the need for an additional costly device. Further development will make it possible to detect moving objects close to the robot, thus predicting possible collision locations. 


\section{Simulation}

A fast and economical way of testing different design concepts is to use simulation techniques with models as realistic as possible. We have developed a simulator package to provide a practical engineering tool to design a full scale robot.

The simulator evaluates an interconnected dynamical time system, consisting of differential equations describing the vehicle motion including the dynamics of the steering mechanism, and difference equations, describing the digital regulators for steering and speed control. The output of the simulation is a list of vehicle positions and orientations. These locations yield input to a CAD program that generates the sweep surface of the robot's motion to check path validity for obstacle avoidance and surface coverage.

In Fig. 4, the two major steps of the simulation process are shown, including path specification and generation of the resulting sweep surface of the vehicle. The simulator CAD package is used to evaluate the feasibility of various vehicle-tool configurations.

\section{Vehicle-ToOl CONFigurations}

The difficulties to find an appropriate vehicle-tool configuration reside in three areas:

* Tool design

* Vehicle surface-load

* Sweep surface of tool and vehicle

Here follows a presentation of necessary functions and requirements in these three areas. In each area we describe various solutions in machines used today and in construction robots presented at different symposiums, followed by discussions of our concepts under evaluation.

\subsection{Tool design}

The common characteristic of the three chosen applications is the presence of rotating tools in contact with the floor and the demand that the tool move with an adequate overlap over the whole working area.

Today, slab finishing is done manually with a single trowel or twin ride-on-trowels with overlapping blades. The grinding machines have single or double rotating discs with grinding attachments.

Tools for a robot must be designed to minimize disturbance forces and torque feedback from the surface via the tool to the vehicle.

Among robots presented at various symposiums there is a wide range of approaches to the tool design [5]-[10]. Shimitzu's FLATKN robot [9] has a slab-finishing tool with three trowels rotating around a center wheel. This design offers good overlap between the trowels, but will have some built-in torque, compensated for by the wheel in contact with the floor.

Our tool system is based on twin trowels rotating in opposite directions. The rotating mechanism is the same in all applications, and will be equipped with: floating blades, finishing blades, grinding tools or cleaning brushes. Rotation speed will be variable, and it will be possible to use overlapping attachments. During the grinding and cleaning mode a vacuum cleaner will collect dust and small objects. 


\subsection{Vehicle surface-load}

The surface load exerted by the robot must not result in remaining tracks or damage to the tooled area. The surface load obviously is most critical during slab finishing [11]. Today, the acceptable surface load is equivalent to the pressure from a worker walking on the concrete when using a trowel. His footprints are easily erased by the trailing trowel.

During the first ten minutes after a surface has been slab-finished it is extremely damageable because of water concentrated in the surface. A wheel following a tool must generate only a fraction of the surface load of a wheel in front of the tool.

Vacuum-treated concrete is frequently used in Sweden. It offers higher surface loads and less waiting time before slab finishing. The waiting time from levelling the concrete to the start of slab finishing is typically 30-60 minutes.

During grinding, the stability of the vehicle is important. Rear wheels roll over a flat and clean surface, resulting in a minimum of location disturbance being transferred to the tool.

While acting in the slab finishing mode it is desirable to use a "tool-following-wheel" concept and in the grinding and cleaning mode the opposite.

\subsection{Sweep surface}

To find a feasible design of vehicle and tool for acceptable surface coverage, it is necessary to identify wasteful and inadequate aspects of the robot's motion. The desired motion pattern to avoid back and forth operations is obtained with the robot processing the surface of the work area in a scanning fashion ("bird-dogging"). The sweep surface of the tool must not contain missed areas and large lateral and turning overlaps. To illustrate these aspects, we will study a twin trowel mounted in the tool-following-wheel fashion on the rear of a vehicle, which is either of tricycle or of differential steering configuration(see Fig. 5). The different aspects are explained below:

- Turning overshoot occurs during turns at the boundary of the working area. With this tool and vehicle concept, the robot is unable to make a turn if it starts turning close to a wall.

- Missed areas occur in the tooled surface during turns at the edges of the field and at obstacles. Because of its shape the tool cannot reach into a corner. The U-turns leave isolated spots and other missed areas, including wedge-shaped sections at the edges of the work area, the sizes of which vary with the turning radius of the robot.

- Lateral overlap means redundant surface coverage between two adjacent passes. The large lateral overlap shown in Fig. 5 is a result of the U-turn manoeuvre. In order to eliminate or minimize the isolated missed area, the turning radius has to be decreased, resulting in an even larger overlap.

- Turning overlap occurs during turns between adjacent passes. As with lateral overlap, it causes double coverage in the work area, time waste, and extra cost.

To eliminate or minimize the above detriments, there are various approches. Kajima's Mark I [5] robot utilizes a tool mounted on the end of a robot arm. The control of the toolpath is a combination of controlling the vehicle path and the position of the tool in the vehicle frame. This solution provides tool-path control that may solve all the overlap and missed area problems listed here, at the cost of a relatively complex design. 
In a fixed tool design, another example is Takenaka's Surf Robo [6]. Good surface coverage is achieved by mainly running the robot in a differential steering mode, with the translation between two adjacent passes accomplished in a tandem drive configuration. The orientation of the drive units are changed while they are in an elevated position.

In our efforts to find an optimal vehicle-tool configuration, evaluation of various designs is underway. In Fig. 6, the structure of a dual drive, dual steer vehicle equipped with twin trowels is shown, along with sweep surfaces resulting from a U-turn at the corner of the work area. The robot follows the prescribed path by tracking it with its two independently inteered and driven rollers (wheels). No turning overshoot and no isolated missed area occur pass along the edge of the work area.

\section{CONCLUSION}

Designing a multipurpose robot for concrete slab finishing, grinding and cleaning involves several engineering disciplines. In phase 1, we have concentrated our efforts on the development of an appropriate onboard controller and evaluation of different navigation configuration.

The built-in adaptability of the control system to various locating devices enables us to use sensors ranging from a low-cost rebar detector to a sophisticated laser range-finder.

The developed path planning algorithms aid the operator during the preparation of a program. It automatically generates a sweep pattern of the area, including obstacle avoidance
and tool overlap.

The dual steer, dual drive vehicle configuration provides good surface coverage but has the disadvantage of undesirable surface loads from the rear wheel while the robot operates in the slab-finishing mode. This problem remains to be solved.

The developed simulation package has proven to be a powerful tool during evaluation of several proposed robot configurations.

The result of the work done so far we hope will enable us to develop a full scale prototype to be tested on a construction site within a year from now.

\section{Acknowledgement}

The authors would like to express their great appreciation to the project leaders Mr. Pär Åmman, The Swedish Construction Federation in Gothenburg, and Mr. Jan-Olof Johansson, Administration and R\&D Manager, Platzer Bygg $A B$, for their devotion in promoting this research project, which is financed by support from The Development Fund Of The Swedish Construction Industry and The Swedish Work Environment Fund. 


\section{REFERENCES}

[1] F. Weiczer, "Development of the Navigation System for a Mobile Robot for Concrete Surface Processing", Proc."Robotikdagarna" ,Linköping, Sweden, May 1991, pp. G1-G10.

[2] J.L. Salagnac, "Structuration Environment and Guidance of a Mobile Robot for Construction Applications", Proc. 5th International Symposium on Robotics in Construction, June 1988, pp. 351-359.

[3] R. Hinkel, T. Knieriemen, E. v. Puttkamer, "MOBOT-III - An Autonomous Mobile Robot for Indoor Applications", Proc. 19th International Symposium on Industrial Robots, 1988, pp. 489-504.

[4] IBEO, Product literature LADAR 2D, IBEO Lasertechnik GmbH, Germany.

[5] M. Saito, N Tanaka, K. Arai and K Banno, "The Development of a Mobile Robot for Concrete Slab Finishing", Proc. 15th International Symposium on Industrial Robots, Sept 1985, pp. 71-78.

[6] K. Kikuchi, S. Furuta and T. Imai, "Development and the Result of Practical Works of Concrete Floor Finishing Robot", Proc. 5th International Symposium on Robotics in Construction, June 1988, pp. 561-569.

[7] N. Tanaka, M. Saito, K. Arai, K. Banno, T Ochi, S. Kikuchi, "The Development of the "Mark II" Mobile Robot for Concrete Slab Finishing", Proc. 5th International Symposium on Robotics in Construction, June 1988, pp. 249-257.

[8] T. Shiokawa and Y. Yamashita, "Navigation/Positioning Control of Mobile Robots for Construction", Proc. 5th International Symposium on Robotics in Construction, June 1988, pp. 589-598.

[9] T. Ueno, Y. Kajioka, H. Sato, J. Maeda, N. Okuyama. "Research \& Development on Robotic Systems for Assembly and Finishing Work", Proc. 5th International Symposium on Robotics in Construction, June 1988, pp. 279-287.

[10] J. Maeda, T. Ueno, T Yoshida, "Development and Application of Cooperative Robots for Construction Work", Proc. 16th International Symposium on Industrial Robots, Oct. 1986, pp. 679-689.

[11] Örjan Petersson, Arne Johansson, "Controlling Trowelling Hardness", CBI Report 1:91, ISSN 0346-8240. 1991. (in Swedish).

[12] J.L. Salagnac, "The functional analysis: An original approach to the robotization of construction tasks", Proc. 6th International Symposium on Robotics in Construction, June 1989, pp, 412-419.

[13] J.L. Crowley, "Asynchronous Control of Translation and Rotation in a Robot Vehicle", Proc. IEEE/RSJ International Workshop on Intelligent Robots and Systems, Tsukuba, Japan, Sept. 1989, pp. 650-655.

[14] W.L. Nelson, I.J. Cox, "Local Path Control for an Autonomous Vehicle", Proc. IEEE International Conf. on Robotics and Automation, Philadelphia, 1988, pp. 1504-1510. 


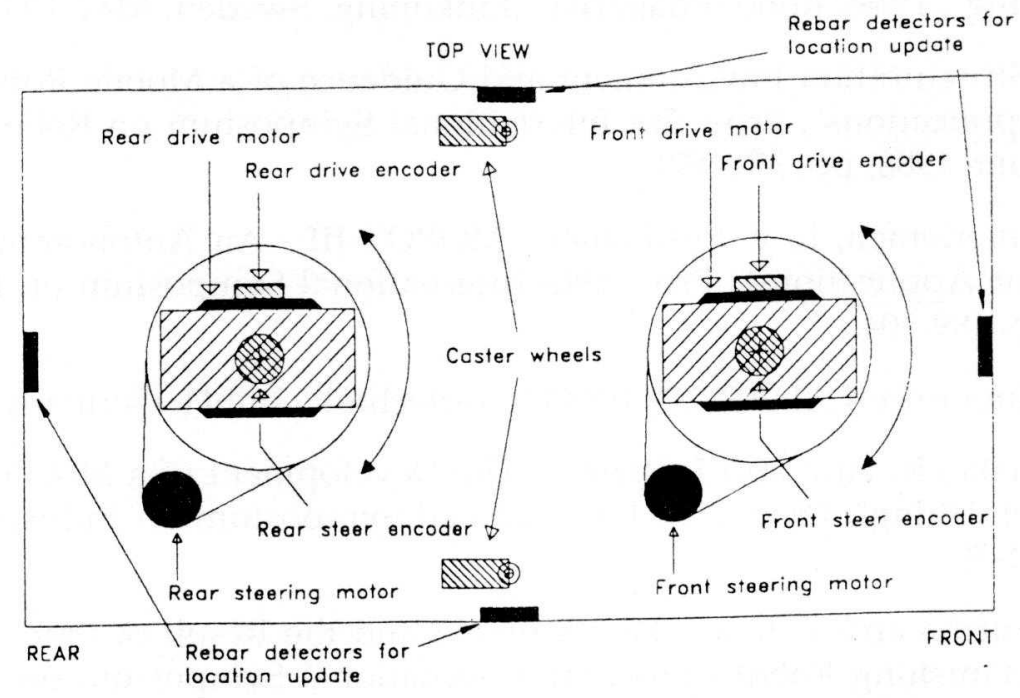

Fig. 1. Steering and drive configuration of the experimental vehicle.

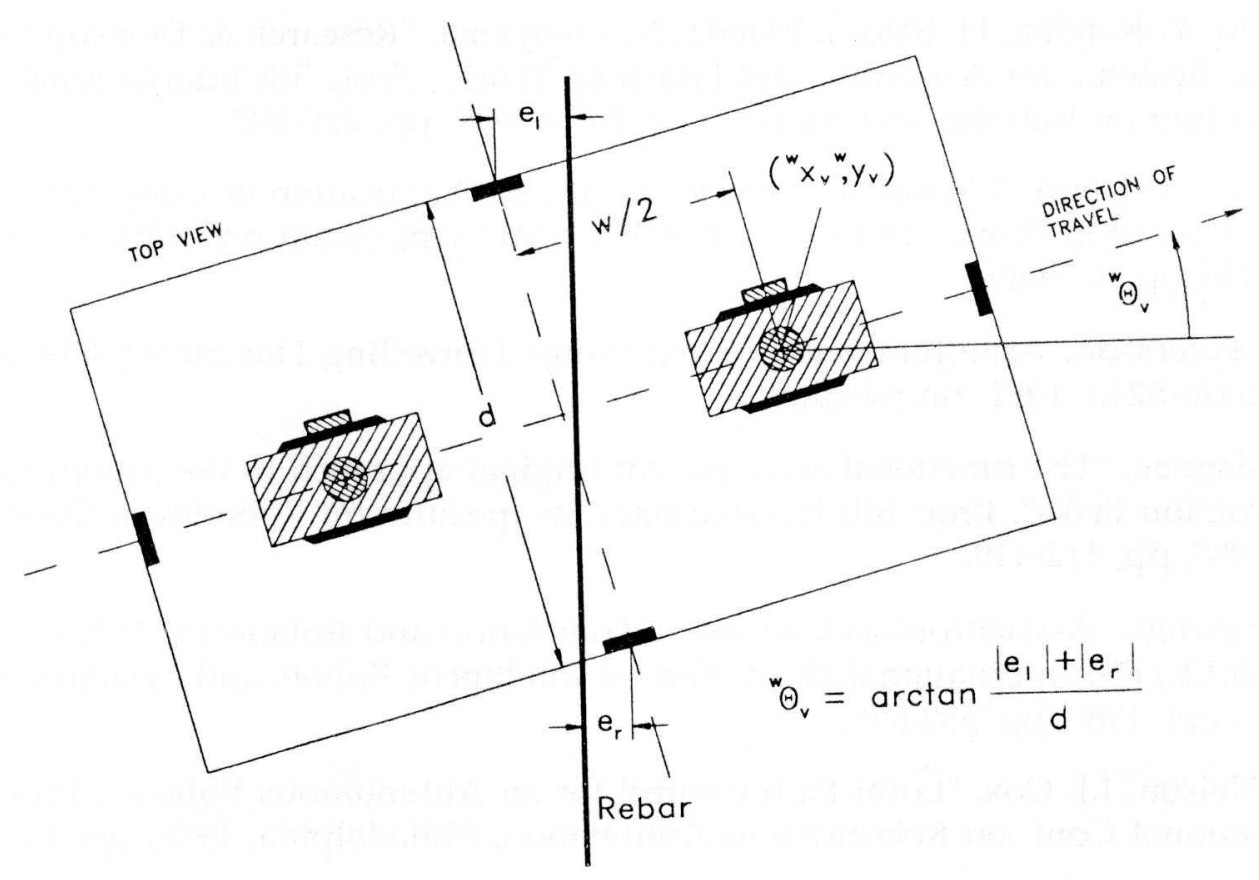

Fig. 2. Example of orientation measurement while passing over a reinforcement-bar. 
L A D A R

AR $\mathrm{GmbH} \& \mathrm{Co} \quad \mathrm{KG}$

Version fuer

ATS Schweden

September 1991

- SCANNING -

F2 Scanbereich

E3 Warnbereich

F4 Profil sp.

INS Sammeln

TAB Grid an/aus

DEL Clear

CR STANDBY

ESC Ende

$-4670 \ldots 7670$

Y : $\quad-5333 \ldots 533$
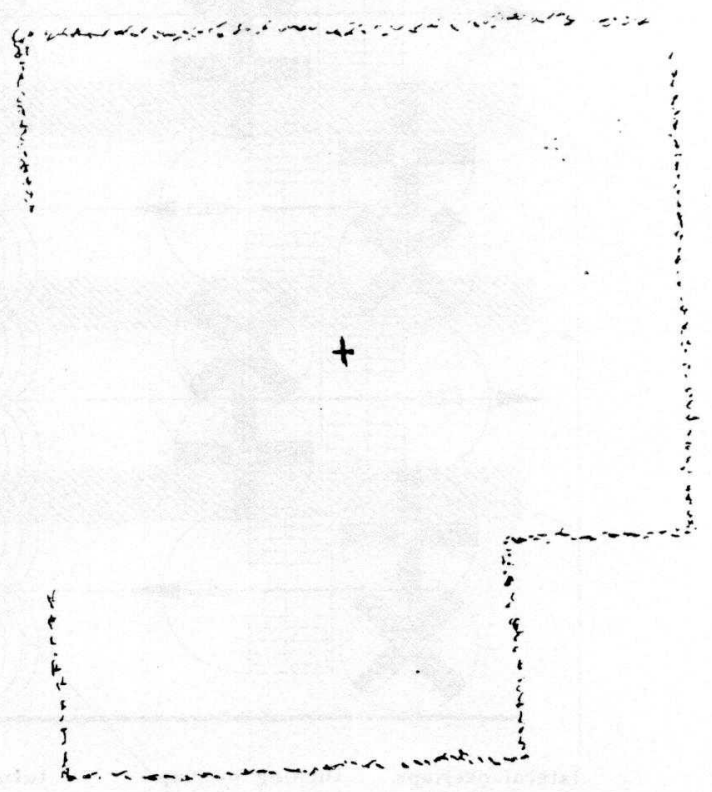

Grid 1000

Recs

[mm]

447

Fig. 3. Example showing a laser scan of the walls around the robot.

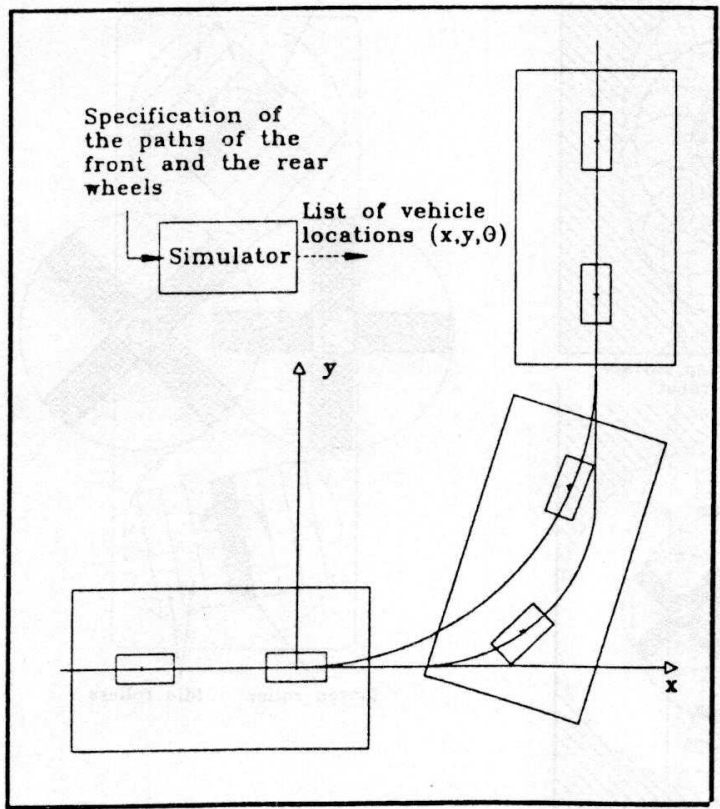

Fig. 4a. Illustration of start and goal locations and path specification for simulation.

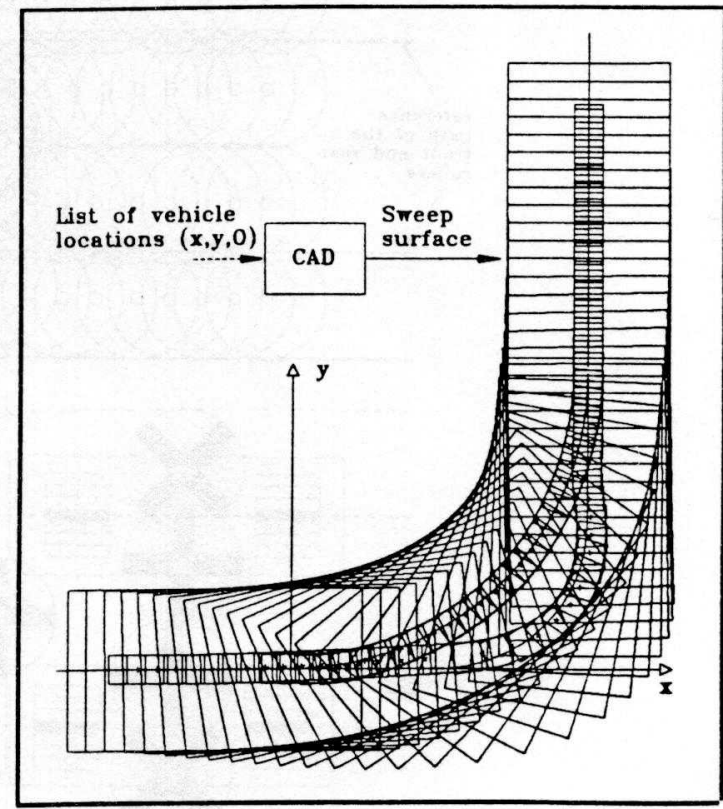

Fig. 4b. CAD generated sweep surface resulting from a simulation. 


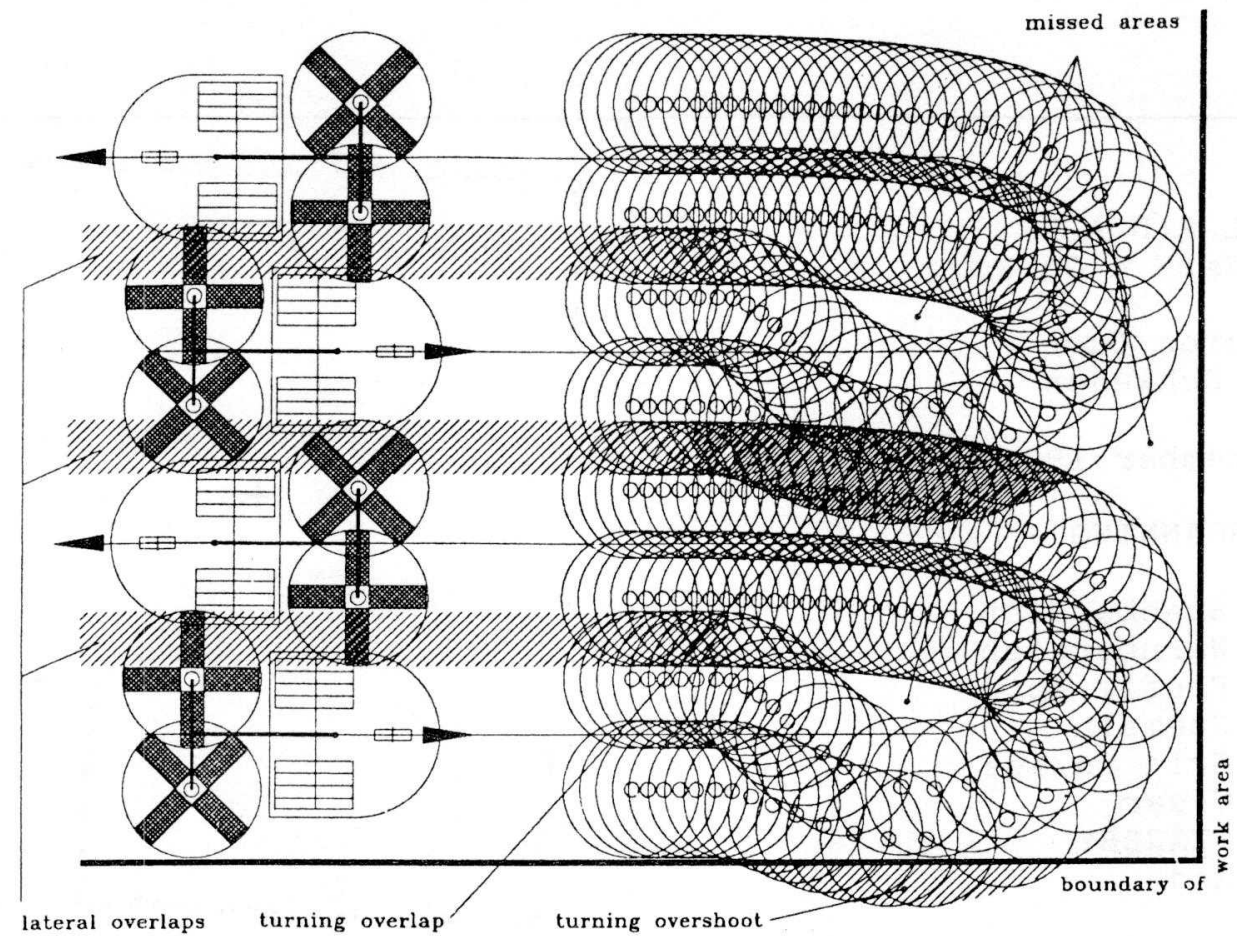

Fig. 5. Simulated robot motion with corresponding sweep surface, illustrating turning overshoot, lateral and turning overlaps, and missed areas.
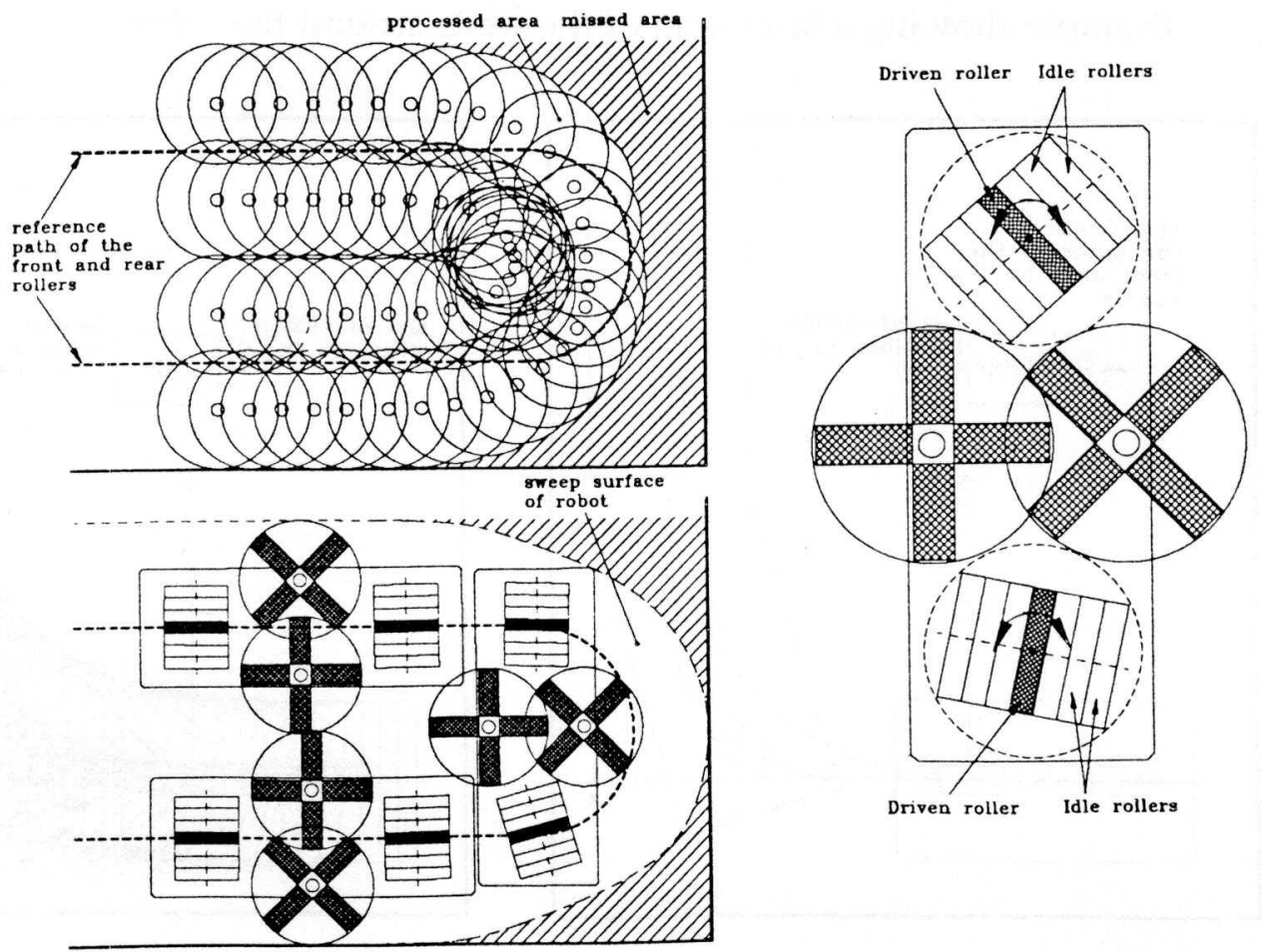

Fig. 6. Structure and sweep surfaces of the dual drive, dual steer vehicle equipped with twin trowels. 